EPJ Web of Conferences 64, 08006 (2014)

DOI: $10.1051 /$ epjconf/ 20146408006

(C) Owned by the authors, published by EDP Sciences, 2014

\title{
Magnetic fields of Herbig Ae/Be stars
}

\author{
S. Hubrig ${ }^{1, a}$, I. Ilyin ${ }^{1}$, M. Schöller ${ }^{2}$, C. R. Cowley $^{3}$, F. Castelli ${ }^{4}$, B. Stelzer ${ }^{5}$, J. -F. Gonzalez ${ }^{6}$, and \\ B. Wolff ${ }^{2}$ \\ ${ }^{1}$ Leibniz-Institut für Astrophysik Potsdam (AIP), An der Sternwarte 16, 14482 Potsdam, Germany \\ ${ }^{2}$ European Southern Observatory, Karl-Schwarzschild-Str. 2, 85748 Garching bei München, Germany \\ ${ }^{3}$ Department of Astronomy, University of Michigan, Ann Arbor, MI 48109-1042, USA \\ ${ }^{4}$ Istituto Nazionale di Astrofisica, Osservatorio Astronomico di Trieste, via Tiepolo 11, 34143 Trieste, Italy \\ ${ }^{5}$ INAF-Osservatorio Astronomico di Palermo, Piazza del Parlamento 1, 90134 Palermo, Italy \\ ${ }^{6}$ Instituto de Ciencias Astronomicas, de la Tierra, y del Espacio (ICATE), San Juan, Argentina
}

\begin{abstract}
We report on the status of our spectropolarimetric studies of Herbig Ae/Be stars carried out during the last years. The magnetic field geometries of these stars, investigated with spectropolarimetric time series, can likely be described by centred dipoles with polar magnetic field strengths of several hundred Gauss. A number of Herbig Ae/Be stars with detected magnetic fields have recently been observed with X-shooter in the visible and the near-IR, as well as with the high-resolution near-IR spectrograph CRIRES. These observations are of great importance to understand the relation between the magnetic field topology and the physics of the accretion flow and the accretion disk gas emission.
\end{abstract}

\section{Introduction}

Magnetic fields are important ingredients of the star formation process (e.g. McKee \& Ostriker 2007 [1]). Models of magnetically driven accretion and outflows successfully reproduce many observational properties of low-mass pre-main sequence stars. Indirect observational evidence for the presence of magnetic fields in these stars is manifested in strong X-ray, FUV, and UV emission (e.g. Feigelson \& Montmerle 1999 [2]). The first detections of magnetic fields in protostars of class I and II sources were obtained using NIR spectrographs and revealed kG fields (e.g. Johns-Krull et al. 2009 [3]). The first magnetic-field maps of T Tauri stars show some systems that have complex fields while some have much simpler dipolar/octupolar fields (e.g. Donati et al. 2008 [4]). Accretion models based on these maps demonstrate the strong dependence of accretion efficiency on both the strength and the geometry of the star's magnetic field.

Fields have also been detected in a dozen Herbig Ae/Be stars (e.g. Hubrig et al. 2009 [5]). Similar to T Tau stars, Herbig Ae/Be stars show clear signatures of surrounding disks as evidenced by a strong infrared excess and are actively accreting material. Current theories are not able to present a consistent scenario of how the magnetic fields in Herbig Ae/Be stars are generated and how these fields interact with the circumstellar environment, consisting of a combination of disk, wind, accretion, and jets. On the other hand, understanding the interaction between the central stars, their magnetic fields, and their

ae-mail: shubrig@aip.de

This is an Open Access article distributed under the terms of the Creative Commons Attribution License 2.0, which permits unrestricted use, distribution, and reproduction in any medium, provided the original work is properly cited. 
protoplanetary disks is crucial for reconstructing the Solar System's history, and to account for the diversity of exo-planetary systems.

\section{Recent studies of the presence of magnetic fields in Herbig Ae/Be stars}

Before 2004, the only magnetic field detection of about $50 \mathrm{G}$ had been reported for the optically brightest $(V=6.5$ ) Herbig Ae star HD 104237 (Donati et al. 1997 [6]), but no further publications confirming this detection existed until recently. Using high-resolution, high signal-to-noise HARPSpol observations (Hubrig et al. 2013 [7]) detected a mean longitudinal magnetic field of the order of $60 \mathrm{G}$. Spectropolarimetric studies from 2004 to 2008 reported the discovery of magnetic fields in seven other Herbig Ae/Be stars (Wade et al. 2005 [8], 2007 [9], Catala et al. 2007 [10], Hubrig et al. 2004 [11], 2006 [12], 2007 [13]). Later on, a study of 21 Herbig Ae/Be stars with FORS 1 revealed the presence of magnetic fields in six additional stars (Hubrig et al. 2009 [5]). More recent studies involved the outbursting binary ZCMa (Szeifert et al. 2010 [14]), the Herbig Ae star HD 101412 with resolved magnetically split lines, and HD $31648=$ MWC 480 (Hubrig et al. 2010 [15], 2011a [16]).

\subsection{Magnetic field versus observed properties}

Spectropolarimetric observations of a sample of 21 Herbig Ae/Be stars observed with FORS 1 have been used to search for a link between the presence of a magnetic field and other stellar properties (Hubrig et al. 2009 [5]). This study did not indicate any correlation of the strength of the longitudinal magnetic field with disk orientation, disk geometry, or the presence of a companion. No simple dependence on the mass-accretion rate was found, but the range of observed field values qualitatively supported the expectations from magnetospheric accretion models with dipole-like field geometries. Both the magnetic field strength and the X-ray emission showed hints of a decline with age in the range of $\sim 2-14 \mathrm{Myr}$ probed by the sample, supporting a dynamo mechanism that decays with age. Furthermore, the stars seemed to obey the universal power-law relation between magnetic flux and $\mathrm{X}$-ray luminosity established for the Sun and main-sequence active dwarf stars.

\subsection{Magnetic field geometry}

Series of mean longitudinal magnetic-field measurements were recently obtained at low resolution with the multi-mode instrument FORS 2 at the VLT for the Herbig Ae/Be stars HD 97048, HD 101412, HD 150193, and HD 176386 (Hubrig et al. 2011b [17]). Magnetic fields of the order of 120-250 G were for the first time detected in these stars a few years ago during our visitor run with FORS 1 in May 2008 (Hubrig et al. 2009 [5]). In our observations, the Herbig Ae/Be stars exhibit a single-wave variation in the longitudinal magnetic field during the stellar rotation cycle. This behaviour is usually considered as evidence for a dominant dipolar contribution to the magnetic field topology. Presently, the Herbig Ae star HD 101412 possesses the strongest longitudinal magnetic field ever measured in any Herbig Ae star, with a surface magnetic field $\langle B\rangle$ up to $3.5 \mathrm{kG}$. HD 101412 is also the only Herbig $\mathrm{Ae} / \mathrm{Be}$ star for which the rotational Doppler effect was found to be small in comparison to the magnetic splitting and several spectral lines observed in unpolarised light at high dispersion are resolved into magnetically split components (Hubrig et al. 2010 [15], 2011b [17]).

To date, magnetic field geometries have been studied for the two SB2 systems HD 200775 (with a B3 primary) and V380 Ori (with a B9 primary) (Alecian et al. 2008 [18], 2009 [19]), and the presumably single stars HD 101412, HD 97048, HD 150193, and HD 176386 (Hubrig et al. 2010 [15], 2011b [17]). As an example, phase diagrams of the magnetic data for the Herbig Ae/Be stars HD 101412 and HD 150193 folded with the determined magnetic/rotation periods are presented in Fig. 1. 

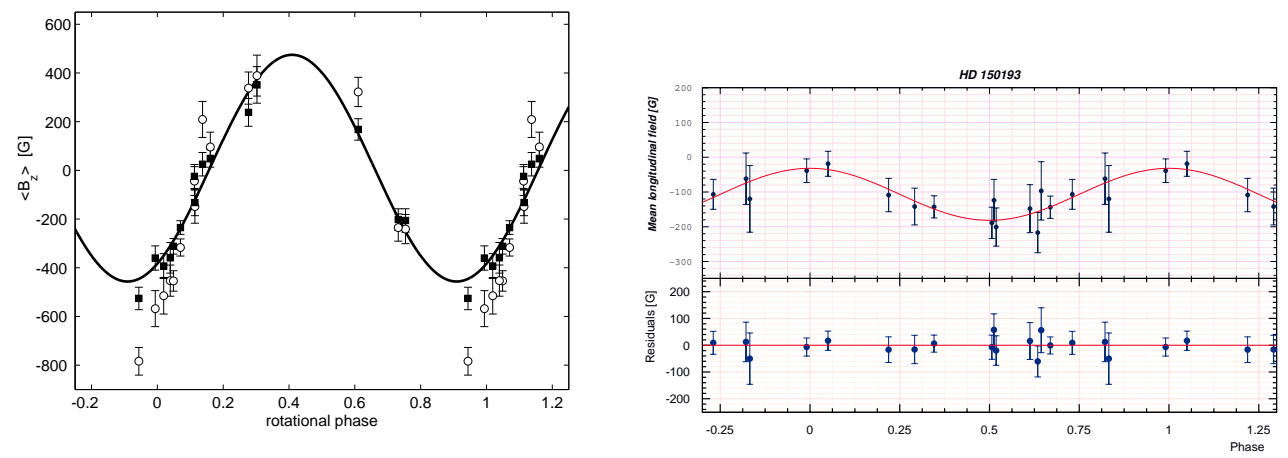

Figure 1. Left panel: Phase diagram of HD 101412 with the best sinusoidal fit for the longitudinal magnetic field measurements using all lines (filled squares) and hydrogen lines (open circles). Right panel: Phase diagram of HD 150193 with the best sinusoidal fit for the longitudinal magnetic field measurements using all lines. The residuals (Observed - Calculated) are shown in the lower panel.

Our magnetic field model for the Herbig Ae star HD 101412 is described by a centered dipole with a polar magnetic field strength $B_{\mathrm{d}}$ between 1.5 and $2 \mathrm{kG}$ and an inclination of the magnetic axis to the rotation axis $\beta$ of $84 \pm 13^{\circ}$ (Hubrig et al. 2011a [16]). The fact that the dipole axis is located close to the stellar equatorial plane is very intriguing in view of the generally assumed magnetospheric accretion scenario that magnetic fields channel the accretion flows towards the stellar surface along magnetic field lines. As was shown in the past (Romanova et al. 2003 [20]), the topology of the channeled accretion critically depends on the tilt angle between the rotation and the magnetic axis. For large inclination angles $\beta$, many polar field lines would thread the inner region of the disk, while the closed lines cross the path of the disk matter, causing strong magnetic braking, which could explain the observed unusually long rotation period of HD 101412 of about 42 days.

Since about $70 \%$ of the Herbig Ae/Be stars appear in binary/multiple systems (Baines et al. 2006 [21]), special care has to be taken in assigning the measured magnetic field to the particular component in the Herbig Ae/Be system. Alecian et al. (2009 [19]) reported on the discovery of a dipolar magnetic field in the Herbig Be star HD 200775, which is a double-lined spectroscopic binary system. However, it should be noted that the magnetic field was discovered not in the component possessing a circumstellar disk and dominating the $\mathrm{H} \alpha$ emission, so that the evolutionary status of the $\mathrm{B} 3$ primary component is yet unclear (Benisty et al. 2013 [22]). Similar to the case of HD 200775, the frequently mentioned discovery of a magnetic field in the Herbig SB2 system HD 72106 (Alecian et al. 2009 [19]) refers to the detection only in the primary component, which is a young main-sequence star, but not in the Herbig Ae secondary (Folsom et al. 2008 [23]). The same uncertainty in the evolutionary status applies to the magnetic field detection in the system V380 Ori reported by Alecian et al. (2009 [19]). The authors detected the presence of a dipole magnetic field of polar strength $2.12 \pm 0.15 \mathrm{kG}$ on the surface of the chemically peculiar primary V380 Ori system. V380 Ori has a spectral type around B9 and has been observed in great detail over many wavelength ranges (e.g., Hamann \& Persson 1992 [24], Rossi et al. 1999 [25], Stelzer et al. 2006 [26]). It has a close infrared companion, with a separation of $0.15^{\prime \prime}$ at PA $204^{\circ}$ (Leinert et al. 1997 [27]). Alecian et al. (2009 [19]) found that the primary in the V380 Ori system is itself a spectroscopic binary with a period of 104 days, with the secondary being a massive T Tauri star. Most recently, Reipurth et al. (2013 [28]) report that V380 Ori is a hierarchical quadruple system with a fourth component at a distance of $8.8^{\prime \prime}$ and position angle $120.4^{\circ}$. Since no periodicity was found in the behaviour of the emissions in hydrogen, helium, cal- 
cium, and oxygen lines (the lines determining the Herbig Ae/Be nature), it is possible that the primary chemically peculiar component with the detected dipolar magnetic field is already at an advanced age and the Herbig Be status of the primary is merely based on the appearance of emission in the above mentioned lines belonging to the secondary T Tau component.

The presence of a magnetic field on the surface of the Herbig Ae star HD 190073 is already known for several years. The first measurement of a longitudinal magnetic field in HD 190073 was published by Hubrig et al. (2006 [12]), indicating the presence of a longitudinal magnetic field $\left\langle B_{\mathrm{z}}\right\rangle=84 \pm 30 \mathrm{G}$ measured on FORS 1 low-resolution spectra at a $2.8 \sigma$ level. Later on this star was studied by Catala et al. (2007 [10]) using ESPaDOnS observations, who confirmed the presence of a weak longitudinal magnetic field, $\left\langle B_{\mathrm{Z}}\right\rangle=74 \pm 10 \mathrm{G}$, at a higher significance level. A few years later a longitudinal magnetic field $\left\langle B_{\mathrm{z}}\right\rangle=104 \pm 19 \mathrm{G}$ was reported by Hubrig et al. (2009 [5]) using FORS 1 measurements. The measurement of the longitudinal magnetic field using archival HARPS observations from May 2011, $\left\langle B_{\mathrm{z}}\right\rangle=91 \pm 18 \mathrm{G}$, fully confirms the presence of a rather stable weak field (Hubrig et al. 2013 [7]). Surprisingly, new observations of this star during July 2011 and October 2012 by Alecian et al. (2013 [29]) detected variations of the Zeeman signature in the LSD spectra on timescales of days to weeks. The authors suggest that the detected variations of Zeeman signatures are the result of the interaction between the fossil field and the ignition of a dynamo field generated in the newly-born convective core. As our recent measurements (Hubrig et al. 2013 [7]) completely contradict those presented by Alecian et al. (2013 [29]), which indicate $\left\langle B_{\mathrm{Z}}\right\rangle=-10 \pm 20 \mathrm{G}$, careful spectropolarimetric monitoring over the next years is important to confirm the reported variability of the magnetic field. Furthermore, since HD 190073 is very likely a binary system (Baines et al. 2006 [21]), special care has to be taken in the interpretation of the magnetic field measurements.

\subsection{CRIRES and X-shooter observations of magnetic Herbig Ae/Be stars}

As mentioned above, all previously studied Herbig Ae/Be stars exhibit a single-wave variation in the longitudinal magnetic field during the stellar rotation cycle. These observations are usually considered as evidence for a dominant dipolar contribution to the magnetic field topology. Magnetospheric accretion theories traditionally consider simple $\sim \mathrm{kG}$ dipolar magnetic fields that truncate the disk and force in-falling gas to flow along the field lines. The assumption of the dominance of dipole fields is usually made for simplicity or due to the lack of available information about the true large-scale magnetic field topology of these stars. Indeed, the recent work of Adams \& Gregory (2012 [30]) shows that high order field components may even play a dominant role in the physics of the gas inflow, as the accretion columns approach the star.

The rather new diagnostic He I $\lambda 1.083 \mu \mathrm{m}$ emission line is considered as probing inflow (accretion) and outflow (winds) in the star-disk interaction region of accreting T Tauri and Herbig Ae/Be stars. The uniqueness of this probe derives from the metastability of this transition and makes it a good indicator of wind and funnel flow geometry (Edwards et al. 2006 [31]). Further, according to Edwards et al., the He I line appears in emission for higher mass accretion rates and in net absorption for lower mass accretion rates. Modeling of this line allowed Gregory et al. (2013, in preparation) for the first time to study the influence of field topologies on the star-disk interaction. Their models use magnetic fields with an observed degree of complexity, as determined via field extrapolation from stellar magnetic maps.

In Fig. 2 we present our recent high-resolution CRIRES observations of the spectral regions containing the He I $\lambda 1.083 \mu \mathrm{m}$ line and the hydrogen recombination line Pa $\gamma$ at $1.094 \mu \mathrm{m}$ over the rotation period of HD 101412 (Hubrig et al. 2012 [33]). The rather strong variation of the line profile of the He I line indicates that the magnetic field of this star is likely more complex than a dipole field. A 

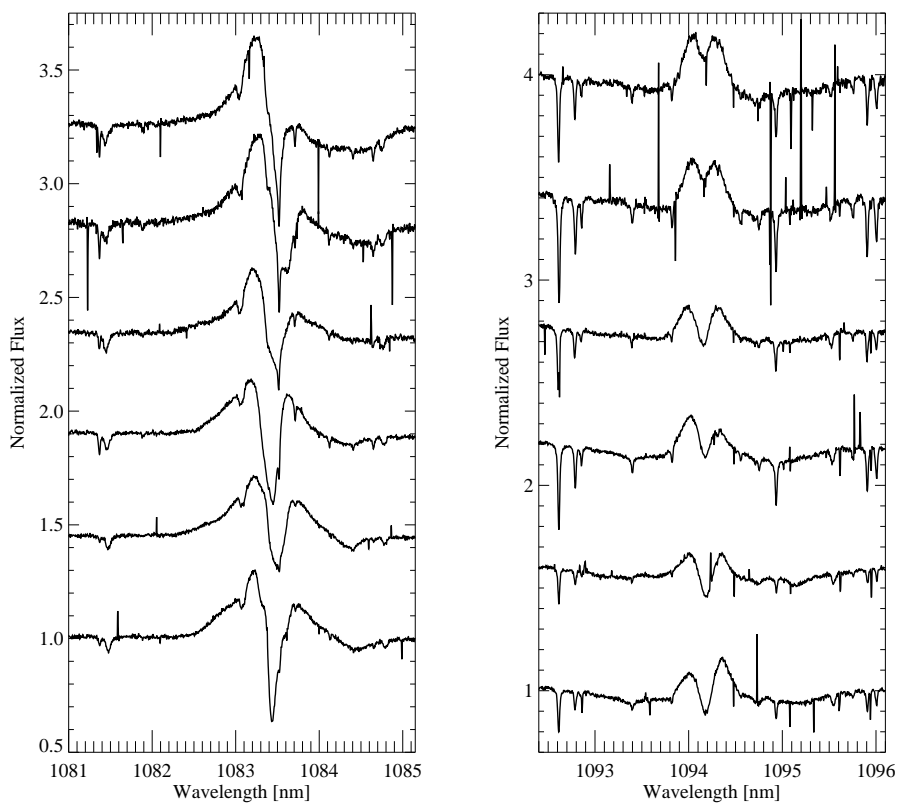

Figure 2. Recent CRIRES observations of HD 101412. Left panel: The variability of the He I $\lambda 1.083 \mu \mathrm{m}$ line profile over the rotation period. Obviously, the field of HD 101412 appears more complex than just a dipole. Right panel: Variations of the hydrogen recombination line Pa $\gamma$ at $1.094 \mu \mathrm{m}$ at the same rotation phases. The Pa $\gamma$ line at $1.094 \mu \mathrm{m}$ is frequently employed for calculating the mass accretion rate in the way presented by Gatti et al. (2008 [32]).

variable behaviour of the $\mathrm{He} \mathrm{I} \lambda 1.083 \mu \mathrm{m}$ line was also discovered in our recent X-shooter spectra of the magnetic Herbig Ae stars HD 190073 and PDS 2 (see Fig. 3).

\section{Discussion}

To understand the magnetospheres of Herbig Ae/Be stars and their interaction with the circumstellar environment presenting a combination of disk, wind, accretion, and jets, the knowledge of the magnetic field strength and topology is indispensable. Progress in understanding the disk-magnetosphere interaction can, however, only come from studying a sufficient number of targets in detail to look for various patterns encompassing this type of pre-main sequence stars.

\section{References}

[1] McKee, C. F., Ostriker, E. C., ARA\&A 45, 565 (2007)

[2] Feigelson, E. D., Montmerle, T., ARA\&A 37, 363 (1999)

[3] Johns-Krull, C. M., Greene, T. P., Doppmann, G. W., Covey, K. R., ApJ 700, 1440 (2009)

[4] Donati, J.-F., Jardine, M. M., Gregory, S. G., et al., MNRAS 386, 1234 (2008) 

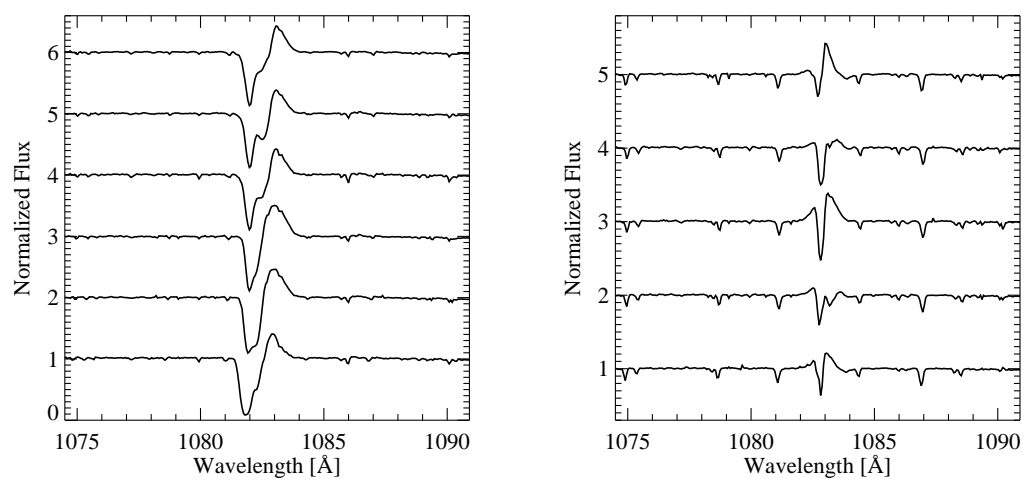

Figure 3. Recent X-shooter observations of the $\mathrm{He} \mathrm{I} \lambda 1.083 \mu \mathrm{m}$ line profile in the magnetic Herbig Ae stars HD 190073 (left panel) and PDS 2 (right panel) at different epochs. The spectra are shifted vertically for clarity.

[5] Hubrig, S., Stelzer, B., Schöller, M., et al., A\&A 502, 283 (2009)

[6] Donati, J.-F., Semel, M., Carter, B. D., et al., MNRAS 291, 658 (1997)

[7] Hubrig, S., Ilyin, I., Schöller, M., Lo Curto, G., Astr. Nachr. in press, also arXiv:1307.0133 (2013)

[8] Wade, G. A., Drouin, D., Bagnulo, S., et al., A\&A 442, L31 (2005)

[9] Wade, G. A., Bagnulo, S., Drouin, D., et al., MNRAS 376, 1145 (2007)

[10] Catala, C., Alecian, E., Donati, J.-F., et al., A\&A 462, 293 (2007)

[11] Hubrig, S., Schöller, M., Yudin, R. V., A\&A 428, L1 (2004)

[12] Hubrig, S., Yudin, R. V., Schöller, M., Pogodin, M. A., A\&A 446, 1089 (2006)

[13] Hubrig, S., Pogodin, M. A., Yudin, R. V., et al., A\&A 463, 1039 (2007)

[14] Szeifert, T., Hubrig, S., Schöller, M., et al., A\&A 509, L7 (2010)

[15] Hubrig, S., Schöller, M., Savanov, I., et al., Astr. Nachr. 331, 361 (2010)

[16] Hubrig, S., Mikulášek, Z., González, J. F., et al., A\&A 525, L4 (2011a)

[17] Hubrig, S., Schöller, M., Ilyin, I., et al., A\&A 536, A45 (2011b)

[18] Alecian, E., Catala, C., Wade, G. A., et al., MNRAS 385, 391 (2008)

[19] Alecian, E., Wade, G. A., Catala, C., et al., MNRAS 400, 354 (2009)

[20] Romanova, M. M., Ustyugova, G. V., Koldoba, A. V., et al., ApJ 595, 1009 (2003)

[21] Baines, D., Oudmaijer, R. D., Porter, J. M., Pozzo, M., MNRAS 367, 737 (2006)

[22] Benisty, M., Perraut, K., Mourard, D., et al., A\&A 555, A113 (2013)

[23] Folsom, C. P., Wade, G. A., Kochukhov, O., et al., MNRAS 391, 901 (2008)

[24] Hamann, F., Persson, S. E., ApJS 82, 285 (1992)

[25] Rossi, C., Errico, L., Friedjung, M., et al., A\&AS 136, 95 (1999)

[26] Stelzer, B., Micela, G., Hamaguchi, K., Schmitt, J. H. M. M., A\&A 457, 223 (2006)

[27] Leinert, C., Richichi, A., Haas, M., A\&A 318, 472 (1997)

[28] Reipurth, B., Bally, J., Aspin, C., et al., AJ in press

[29] Alecian, E., Neiner, C., Mathis, S., et al., A\&A 549, L8 (2013)

[30] Adams, F. C., Gregory, S. G., ApJ 744, 55 (2012)

[31] Edwards, S., Fischer, W., Hillenbrand, L., Kwan, J., ApJ 646, 319 (2006)

[32] Gatti, T., Natta, A., Randich, S., et al., A\&A 481, 423 (2008)

[33] Hubrig, S., Castelli, F., González, J. F., et al., A\&A 542, A31 (2012) 